\title{
Extensions and applications of Fuller's theorem on periodic points
}

\author{
H. T. KU, M. C. KU and L. N. MANN
}

(Received May 24, 1993)

\begin{abstract}
If $f: X \rightarrow X$ is a continuous function, $x \in X$ is called a periodic point if some iterate $f^{k}$ of $f$ leaves $x$ fixed. In $1953 \mathrm{~F}$. B. Fuller proved a basic result for the existence of periodic points. He showed that if $X$ is a compact absolute neighborhood retract with non-zero Euler characteristic and if $N(X)$ is the larger of the sums of the odd and even real Betti numbers of $X$, then for any homeomorphism $f$ on $X, f^{k}$ has a fixed point for some $k, 1 \leq k \leq N(X)$. Actually Fuller's result holds for any space $X$ for which the Lefschetz Fixed Point Theorem is valid.

In this paper the authors first establish a number of extensions of the Fuller Theorem. They next consider $G^{-}$spaces and extend results of Mann on periodic points and invariant orbits. In the last section they turn to the Riemannian category using the volume function on the orbit space to obtain additional results and examples on periodic points and invariant orbits for groups of isometries. They conclude the paper by using the Fuller Theorem, together with a result of Kobayashi relating the Lefschetz number of an isometry and the Euler characteristic of its fixed point set, to establish a new result on the existence of common periodic points for commuting isometries.
\end{abstract}

\section{Introduction}

For a compact space $X$, let $b_{q}(X)$ denote the $q^{\text {th }}$ real Betti number of $X$, i. e., the dimension of the vector space $H^{q}(X ; R)$, and $\chi(X)$ the associated Euler characteristic. If $f: X \rightarrow X$ is a continuous function, $x \in$ $X$ is called a periodic point if some iterate $f^{k}$ of $f$ leaves $x$ fixed. In 1953 F. B. Fuller [7] proved the following basic result for the existence of periodic points. We use the formulation in [5].

THEOREM 1.1. (Fuller) Let $X$ be a compact ANR (absolute neighborhood retract) such that $\chi(X) \neq 0$. Let $N(X)$ be the larger of the two integers 


$$
\sum_{q \text { odd }} b_{q}(X) \text { and } \sum_{q \text { even }} b_{q}(X)
$$

If $f: X \rightarrow X$ is any homeomorphism, then $f^{k}$ has a fixed point for some $k$, $1 \leq k \leq N(X)$.

Actually Fuller's result holds for any space $X$ for which the Lefschetz Fixed Point Theorem is valid [5] as the proof proceeds by showing that the Lefschetz number of some iterate $f^{k}, k \leq N(X)$, is non-zero.

In Section 2 we establish a number of extensions of the Fuller Theorem. In Section 3 we consider $G$-spaces and extend results of Mann [14] on periodic points and invariant orbits. We turn in Section 4 to the Riemannian category using the volume function on the orbit space to obtain additional results and examples on periodic points and invariant orbits. We conclude by using the Fuller Theorem, together with a result of Kobayashi [12] relating the Lefschetz number of an isometry and the Euler characteristic of its fixed point set, to establish a result on the existence of common periodic points for commuting isometries.

\section{Extensions of the Fuller Theorem}

For a locally compact space $X$ we will use Alexander-Spanier cohomology with compact supports; our coefficient group will be the reals $R$. In most cases, however, $X$ will be compact.

A continuous self-map $f$ of $X$ induces homomorphisms

$$
f^{* q}: H^{q}(X) \rightarrow H^{q}(X) .
$$

Define

$$
\chi_{f}(X)=\sum_{q=0}^{\infty}(-1)^{q} \operatorname{dim} \operatorname{Im} f^{* q},
$$

and

$$
N_{f}(X)=\max \left[\sum_{q \text { odd }} \operatorname{dim} \operatorname{Im} f^{* q}, \sum_{q \text { even }} \operatorname{dim} \operatorname{Im} f^{* q}\right] .
$$

Compare $\chi_{f}(X)$ with the Fuller Index $F(f)$ as defined in [6, p. 26]. Also note that if $f$ is a homeomorphism, $\chi_{f}(X)=\chi(X)$ and $N_{f}(X)=N(X)$.

We now suppose $M$ is a closed connected oriented n-manifold and $g$ : $M \rightarrow M$ continuous. Define

$$
g_{*}^{q}: H^{q}(M ; R) \rightarrow H^{q}(M ; R)
$$

by 


$$
\begin{aligned}
& \left\langle g_{*}^{q} \alpha \cup \beta,[M]\right\rangle=\left\langle\alpha \cup g^{*(n-q)} \beta,[M]\right\rangle, \alpha \in H^{q}(M ; R), \\
& \beta \in H^{n-q}(M ; R) .
\end{aligned}
$$

The following result for deRham cohomology is contained in [8].

LEMMA 2.1. If $\operatorname{deg} g \neq 0$,

$$
g_{*}^{q}=(\operatorname{deg} g)\left(g^{* q}\right)^{-1} \text {. }
$$

PROOF.

$$
\begin{aligned}
\left\langle g_{*}^{q} g^{* q} \alpha \cup \beta,[M]\right\rangle & =\left\langle g^{* q} \alpha \cup g^{*(n-q)} \beta,[M]\right\rangle \\
& =\left\langle g^{* n}(\alpha \cup \beta),[M]\right\rangle \\
& =\left\langle\alpha \cup \beta, g_{* n}[M]\right\rangle \\
& =\langle\alpha \cup \beta,(\operatorname{deg} g)[M]\rangle \\
& =\langle(\operatorname{deg} g) \alpha \cup \beta,[M]\rangle .
\end{aligned}
$$

But $H^{q}(M ; R) \times H^{n-q}(M ; R) \rightarrow R \quad$ where $(\alpha, \beta) \rightarrow\langle\alpha \cup \beta,[M]\rangle$ is nondegenerate so

$$
g_{*}^{q} g^{* q}=(\operatorname{deg} g)(\text { Identity }) .
$$

LEMMA 2.2. If $\operatorname{deg} g \neq 0$ and

$$
f^{* q} \circ g^{* q}=g^{* q} \circ f^{* q} \text {, all } q \text {, }
$$

then

$$
\left(f^{k}\right)^{* q} \circ\left(g_{*}^{k}\right)^{q}=\left(f^{* q} \circ g_{*}^{q}\right)^{k} \text {, all } k \text { and all } q .
$$

PROOF. It suffices to show that

$$
f^{* q} \circ g_{*}^{q}=g_{*}^{q} \circ f^{* q} \text {. }
$$

But

$$
\begin{aligned}
f^{* q} \circ g_{*}^{q} & =f^{* q} \circ(\operatorname{deg} g)\left(g^{* q}\right)^{-1} \\
& =(\operatorname{deg} g)\left(g^{* q}\right)^{-1} \circ f^{* q} \\
& =g_{*}^{q} \circ f^{* q} .
\end{aligned}
$$

We are now in a position to generalize Fuller's Theorem to a pair of continuous maps $f, g: M \rightarrow M$. Define the Lefschetz number of the pair $(f, g)$ by

$$
L_{f, g}=\sum_{q=0}^{n}(-1)^{q} \operatorname{Tr}\left(f^{* q} \circ g_{*}^{q}\right) .
$$

The following result is well-known [15].

THEOREM 2.3. (Lefschetz Coincidence Theorem) If $L_{f, g} \neq 0$, then 
there exists $x \in M$ such that

$$
f(x)=g(x) \text {. }
$$

For $f, g: M \rightarrow M$ also define

$$
\chi_{f, g}(M)=\sum_{q=0}^{n}(-1)^{q} \operatorname{dim} \operatorname{Im}\left(f^{* q} \circ g_{*}^{q}\right)
$$

and

$$
N_{f, g}(M)=\max \left[\sum_{q \text { odd }} \operatorname{dim} \operatorname{Im}\left(f^{* q} \circ g_{*}^{q}\right), \sum_{q \text { even }} \operatorname{dim} \operatorname{Im}\left(f^{* q} \circ g_{*}^{q}\right)\right] .
$$

Observe that for $g=$ identity, $\chi_{f, g}(M)$ and $N_{f, g}(M)$ reduce to $\chi_{f}(M)$ and $N_{f}(M)$ respectively as previously defined.

THEOREM 2.4. (Fuller Coincidence Theorem). Suppose $f, g$ are continuous self-maps of a closed connected oriented $n$-manifold $M$. If $\chi_{f, g}(M) \neq 0$, $\operatorname{deg} g \neq 0$, and

$$
f^{* q} \circ g^{* q}=g^{* q} \circ f^{* q} \text {, all } q,
$$

then there exists $x \in M$ such that

$$
f^{k}(x)=g^{k}(x)
$$

for some $k, 1 \leq k \leq N_{f, g}(M)$.

PRoOF. We show $L_{f^{k}, g^{k}} \neq 0$ for some $k \leq N_{f, g}(M)$ and apply Theorem 2.3. Suppose, on the contrary, that

$$
L_{f^{k}, g^{k}}=0, k=1,2, \ldots, \quad N_{f, g}(M) .
$$

We shall prove $\chi_{f, g}(M)=0$ which contradicts our hypothesis.

Let $\lambda_{q, j}, j=1,2, \ldots i_{q}$, be the non-zero eigenvalues of $f^{* q} \circ g_{*}^{q}$ where

$$
i_{q}=\operatorname{dim} \operatorname{Im}\left(f^{* q} \circ g_{*}^{q}\right) \text {. }
$$

Then

$$
\operatorname{Tr}\left[\left(f^{* q} \circ g_{*}^{q}\right)^{k}\right]=\sum_{j=1}^{i q} \lambda_{q, j}^{k}
$$

From our restrictions on $f$ and $g$ and Lemma 2.2, we have,

$$
\operatorname{Tr}\left[\left(f^{k}\right)^{* q} \circ\left(g_{*}^{k}\right)^{q}\right]=\operatorname{Tr}\left[\left(f^{* q} \circ g_{*}^{q}\right)^{k}\right] .
$$

Since $L_{f^{k}, g^{k}}=0$, it follows from (2) and (3) that 


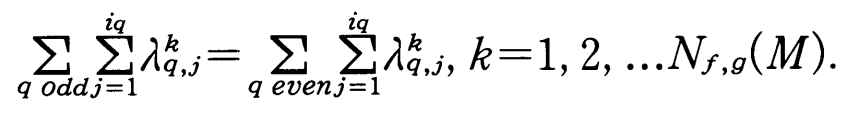

Now using the same argument as presented in $[5, \mathrm{p} .46]$ in the proof of the Fuller Theorem, we can argue that

$$
\sum_{q \text { odd }} i_{q}=\sum_{q \text { even }} i_{q} \text { or } \chi_{f, g}(M)=0 .
$$

REMARK 2.5. Theorem 2.4 is similar to Theorem 23 of Halpern in [9]. In both cases some type of commutativity is assumed. If we take $g$ =identity, we obtain a result similar to Theorem 5.5 in [6]. Actually we can relax the hypothesis of 2.4. Since $\operatorname{deg} g \neq 0, g^{* q}$ is an isomorphism for all $q$ and $N_{f, g}(M)=N_{f}(M)$. Moreover $\chi_{f, g}(M)=(\operatorname{deg} g) \chi_{f}(M)$, so $\chi_{f}(M) \neq 0$ implies $\chi_{f, g}(M) \neq 0$.

We have various immediate consequences of Theorem 2.4.

COROLlary 2.6. Suppose $f, g$ are continuous self-maps of a closed connected oriented manifold $M$. If $\chi_{f, g}(M) \neq 0$, deg $g \neq 0$, and $f$ is homotopic to $g$, then there exists $x \in M$ such that

$$
f^{k}(x)=g^{k}(x)
$$

for some $k, \quad 1 \leq k \leq N_{f, g}(M)$.

COROLlARY 2.7. Suppose $f, g$ are continuous self-maps of a closed connected oriented manifold $M$. If $\chi_{f}(M) \neq 0$ and $g$ is homotopic to the identity, then there exists $x \in M$ such that

$$
f^{k}(x)=g^{k}(x)
$$

for some $k, 1 \leq k \leq N_{f}(M)$.

PROOF. If $g$ is homotopic to the identity, deg $g=1 \neq 0$ and $g^{* q}=$ identity. Hence

$$
\chi_{f, g}(M)=\chi_{f}(M) \neq 0
$$

and

$$
N_{f, g}(M)=N_{f}(M) .
$$

The reader is referred to the beginning of Section 3 for the terminology involving transformation groups in the next several consequences of 2. 4 .

COROLlARY 2.8. Suppose $G$ is a compact connected Lie group acting effectively on a closed connected oriented manifold $M$. If $f$ is a 
continuous self-map of $M$ with $\chi_{f}(M) \neq 0$, then for any $g \in G$, there exists $x \in M$ such that

$$
f^{k}(x)=g^{k}(x)
$$

for some $k, 1 \leq k \leq N_{f}(M)$.

PROOF. If $g \in G, g$ is homotopic to the identity. Clearly if $f$ is equivariant, we can remove the condition that $G$ be connected.

COROLlary 2.9. Suppose $G$ is a compact Lie group acting effectively on a closed connected oriented manifold $M, \chi(M) \neq 0$. If $f, g \in G$, there exists $x \in M$ such that

$$
f^{k}(x)=g^{k}(x)
$$

for some $k$,

$$
1 \leq k \leq\left|G / G^{0}\right|
$$

where $G^{0}$ denotes the identity component of $G$, and $\left|G / G^{0}\right|$, the order of the finite group $G / G^{0}$.

Moreover, letting $[f],[g]$ denote the coset classes in $G / G^{0}$,

i ) If $[f]=[g], k=1$;

ii ) If $[f][g]=[g][f], 1 \leq k \leq \min \left\{\left|G / G^{0}\right|, N(M)\right\}$.

PROOF. Since $f$ and $g$ are homeomorphisms,

$$
\chi_{f, g}(M)=\chi(M) \neq 0 \text { and } N_{f, g}(M)=N(M) .
$$

Let $m=\left|G / G^{0}\right|$. Then

$$
\left[f^{m}\right]=\left[g^{m}\right]=\text { identity element of } G / G^{0} \text {. }
$$

Hence

$$
\left(f^{k}\right)^{* q}=\left(g^{k}\right)^{* q}=\text { identity }
$$

for $k=m, q \geq 0$.

If $[f]=[g]$,

$$
\left(f^{k}\right)^{* q}=\left(g^{k}\right)^{* q}
$$

for $k=1, q \geq 0$. Thus in either case by 2.1 we have,

$$
\begin{aligned}
L_{f^{k}, g^{k}} & =(\operatorname{deg} g) \sum_{q \geq 0}(-1)^{q} \operatorname{Tr}\left[\left(f^{k}\right)^{* q} \circ\left(\left\{f^{k}\right\}^{* q}\right)^{-1}\right] \\
& =(\operatorname{deg} g) \chi(M) \neq 0 .
\end{aligned}
$$


Hence by 2.3, there exists $x \in M$ such that

$$
f^{k}(x)=g^{k}(x) \text {. }
$$

Finally if $[f][g]=[g][f]$,

$$
f^{* q} \circ g^{* q}=g^{* q} \circ f^{* q}, \text { all } q \geq 0,
$$

and we may apply 2.4 to conclude

$$
k \leq N_{f, g}(M)=N(M) \text {. }
$$

COROLlary 2.10. Suppose $S^{n}, n$ even, is the unit sphere in $R^{n+1}$ with standard metric. If $f, g: S^{n} \rightarrow S^{n}$ are isometries, there exists $x \in S^{n}$ such that

$$
f^{k}(x)=g^{k}(x)
$$

for some $k, k=1$ or 2 .

Proof. Note $f, g \in I \operatorname{som}\left(S^{n}\right)=O(n+1)$ and $N\left(S^{n}\right)=2$. Actually $k=$ 1 if $f, g \in S O(n+1)$ or $f, g \notin S O(n+1)$.

\section{Periodic points and invariant orbits of equivariant maps}

Associated with a $G$-space $X$ are basic objects such as the fixed point set $F(G, X)$, the orbit space $X / G$, and the orbits themselves. In [14] Mann made observations concerning the existence of points in $F(G, X)$ which are left fixed and orbits which are left invariant by some interate $f^{k}$ of an equivariant self-map $f$ of $X$. In this section we continue such investigations.

We begin with some background material on topological transformation groups. Roughly speaking $(X, G)$ is called a $G^{-}$space if $X$ is a topological space, $G$ is a topological group, and $G$ acts on $X$ as a group of homeomorphisms [3]. The fixed point set $F(G, X)$ consists of those points of $X$ left fixed by each $g \in G$, the orbit $G(x)$ of $x \in X$ is the subset $\{g(x): g \in G\}$, and the orbit space $X / G$, which we denote often simply by $X^{*}$, is the space of orbits under the decomposition topology. $\pi: X \rightarrow X^{*}$ denotes the natural projection.

The isotropy subgroup $G_{x}$ of $G$ at $x$ is the subgroup $\{g \in G: g(x)=x\}$; isotropy subgroups of points on the same orbit are mutually conjugate. The action of $G$ on $X$ is called effective if $\bigcap_{x \in X} G_{x}$ is simply the identity element of $G$. In a natural way $G(x)$ may be identified with the left coset space $G / G_{x}$. A continuous map $f: X \rightarrow X$ is called equivariant if $f(g(x))=g(f(x))$, all $g \in G$ and $x \in X$. 
We will be considering effective $G$-spaces $(X, G)$ where $X$ is a closed $n$-manifold $M$ and $G$ is a compact connected Lie group. An orbit $G(x)$ is called a principal orbit if $G(x)$ is of maximal dimension among all orbits and $G_{x}$ has a minimal number of components among all orbits of maximal dimension. The associated isotropy subgroup is called a principal isotropy subgroup and it follows that any two principal isotropy subgroups are conjugate. It is known [3;IX] that the union of the principal orbits forms a connected open dense subset of $M$. Orbits which are not principal are of 2 types, exceptional (orbits of maximal dimension) and singular (orbits of lower dimension). We let $S(G, M)$ denote the union of the singular orbits.

THEOREM 3.1. Suppose $\chi(G(x))=0$ for a principal orbit $G(x)$ or $\chi\left(M^{*}-S(G, M)^{*}\right)=0$, then

$$
\chi(M)=\chi(S(G, M)) .
$$

Proof. Since $\operatorname{dim} S(G, M) \leq n-2, M-S(G, M)$ is connected $[3 ; \mathrm{IX}]$. If there are no exceptional orbits in $M-S(G, M)$, we have the fibration:

$$
G / H \rightarrow M-S(G, M) \stackrel{\pi}{\rightarrow} M^{*}-S(G, M)^{*},
$$

where $H$ is a principal isotropy subgroup. Now the Leray sheaf $H^{*}(G / H$; $R)$ over $M^{*}-S(G, M)^{*}$ is constant since $G$ acts trivially on $H^{*}(G / H$; $R)[2 ; \mathrm{VIII}]$. Hence by $[2 ; \mathrm{IX}]$,

$$
\left.\chi(M-S(G, M))=\chi(G / H) \cdot \chi\left(M^{*}-S(G, M)^{*}\right)\right)
$$

and it follows from our hypothesis that

$$
\chi(M-S(G, M))=0 .
$$

If, on the other hand, there are exceptional orbits, let $G / K$ be such an orbit with $K \supset H$. Then $K / H$ is a finite set and we have the fibration:

$$
K / H \rightarrow G / H \rightarrow G / K .
$$

By the Vietoris-Begle mapping theorem, we can argue that

$$
H^{*}(G / K ; R) \cong H^{*}(G / H ; R),
$$

and establish (2) via the spectral sequence of the continuous map $\pi$ instead of the spectral sequence of the fibration. Hence, in any case, we have, 


$$
\chi(M-S(G, M))=0 .
$$

The result now follows from the exact sequence of the pair $(M, S(G, M))$ using Alexander-Spanier cohomology with compact supports.

THEOREM 3.2. Suppose $f: M \rightarrow M$ is an equivariant homeomorphism, $G(x)$ is a principal orbit, and one of the following conditions hold:

i ) $\chi(M) \neq 0$, and $\chi(G(x))=0$ or

$$
\chi\left(M^{*}-S(G, M)^{*}\right)=0 \text {; }
$$

ii ) $\chi(M)=0, \chi(G(x)) \neq 0$, and $\chi\left(M^{*}-S(G, M)^{*}\right) \neq 0$.

Assume, moreover, that the Lefschetz fixed point theorem is valid for $S(G$, $M)$. Then there is a singular orbit $G(y)$ such that every point of $G(y)$ is a periodic point of $f$.

PROOF. Under ( i ), it follows from 3.1 that $\chi(S(G, M))=\chi(M) \neq 0$. Under (ii) it follows from (2) in the proof of 3.1 that $\chi(M-S(G, M)) \neq$ 0 . Hence $\chi(S(G, M)) \neq \chi(M)=0$. Since the Lefschetz fixed point theorem is assumed valid for $S(G, M)$, we can apply the Fuller Theorem to $f$ : $S(G, M) \rightarrow S(G, M)$ and obtain the result.

COROLlary 3.3. Suppose $f: M \rightarrow M$ is an equivariant homeomorphism and $G$ acts on $M$ with principal orbits of codimension one with $M^{*}$ a closed interval. If one of the following conditions hold:

i ) $\chi(M) \neq 0$.

ii ) $\chi(G(x)) \neq 0$, where $G(x)$ is a principal orbit,

then there is a singular orbit $G(y)$ such that every point of $G(y)$ is a periodic point of $f$.

Proof. Since $\chi\left(M^{*}-S^{*}(G, M)^{*}\right) \neq 0$, the result follows from 3.2. Note since $\operatorname{dim} M=\operatorname{dim} G(x)+1, \chi(M) \neq 0$ implies $\chi(G(x))=0$. Compare 3.3 with Theorem 4. 2. of [14].

COROLlary 3.4. Suppose $f: M \rightarrow M$ is an equivariant homeomorphism and $G$ acts on $M$ with principal orbits of codimension two and with at least one singular orbit. Suppose $H_{1}(M ; Z)=0$, where $Z$ denotes the coefficient group of integers, and one of the following conditions hold:

i ) $\chi(M) \neq 0$ and $\chi(G(x))=0$, where $G(x)$ is a principal orbit,

ii ) $\chi(M)=0$ and $\chi(G(x)) \neq 0$. 
Then there is a singular orbit $G(y)$ such that every point of $G(y)$ is a periodic point of $f$.

ProOF. It is known that $M^{*}$ is a 2-disk with $S(G, M)^{*}=\partial M^{*}$ [4], so that $\chi\left(M^{*}-S(G, M)^{*}\right)=1 \neq 0$. The result now follows from 3.2.

EXAMPLE 3.5. Let $G=S O\left(p_{1}\right) \times \cdots \times S O\left(p_{k}\right) \times K, p_{i} \geq 2$, be a compact connected Lie group acting effectively on $M=S^{p_{1}}(1) \times \cdots \times S^{p_{k}}(1) \times K / H$, where $K$ acts transitively on $K / H$ and each $S O\left(p_{i}\right)$ acts on the unit sphere $S^{p_{i}}(1)$ via the standard action which has two fixed points $\left\{a_{i}, b_{i}\right\}$, and principal orbits $S^{p_{i}-1}\left(\theta_{i}\right), 0<\theta_{i}<\pi$. There are no exceptional orbits and the singular orbits have the form:

$$
G(y)=V_{1} \times \cdots \times V_{k} \times K / H,
$$

where

$$
V_{i}=a_{i}, b_{i} \text { or } S^{p_{i}-1}\left(\theta_{i}\right), 0<\theta_{i}<\pi,
$$

with at least one $i$ where $V_{i}=a_{i}$ or $b_{i}$.

Furthermore

$$
M^{*}=\left\{\left(\theta_{1}, \theta_{2}, \ldots, \theta_{k}\right): 0 \leq \theta_{i} \leq \pi ; i=1,2, \ldots, k\right\}
$$

and

$$
\partial M^{*}=S(G, M)^{*} \text {. }
$$

We apply 3.2. If $f: M \rightarrow M$ is an equivariant homeomorphism, then

i ) If $\chi(M) \neq 0$, there exists a singular orbit $G(y)$ such that every point in $G(y)$ is a periodic point of $f$. In fact, there is an orbit $G(y) \approx$ $K / H$ with this property.

ii ) If $\chi(G(x)) \neq 0$ for a principal orbit $G(x)$, the same conclusion follows.

EXAMPLE 3.6. Let $S O\left(p_{1}+1\right) \times \cdots \times S O\left(p_{k}+1\right), p_{i} \geq 1$, act diagonally on $R^{p_{1}+1} \times \cdots \times R^{p_{k+1}}=R^{n+k}, n=p_{1}+\cdots+p_{k}$. We have an induced action on the unit sphere $S^{n+k-1}(1)$ in $R^{n+k}$. Finally let

$$
G=S O\left(p_{1}+1\right) \times \cdots \times S O\left(p_{k}+1\right) \times K,
$$

$K$ a compact connected Lie group, and

$$
M=S^{n+k-1}(1) \times K / H .
$$

The orbit space $M^{*}$ of the action of $G$ on $M$ is given by the $(k-1)$-disk, 


$$
M^{*}=\left\{\left(\theta_{1}, \theta_{2}, \ldots, \theta_{k}\right): \theta_{i} \geq 0 \text { and } \theta_{1}^{2}+\cdots+\theta_{k}^{2}=1\right\} .
$$

The orbits are of the form:

$$
G(z)=S^{p_{1}}\left(r_{1}\right) \times \cdots \times S^{p_{k}}\left(r_{k}\right) \times K / H,
$$

where $r_{1}^{2}+\cdots+r_{k}^{2}=1, r_{i} \geq 0$. (Here we assume $S^{p_{i}}(0)$ is a point). Now $G(z)$ is a singular orbit if at least one $r_{i}=0$. If follows that

$$
S(G, M)^{*}=\partial M^{*}
$$

and we can apply 3.2 to this action.

\section{Isometries}

In this section we turn to the Riemannian category where our $G$-space $(M, G)$ is a compact connected Lie group $G$ acting effectively as a group of isometries on a closed connected Riemannian $n$-manifold $M$. We denote $\pi(G(x))$ by $x^{*}$ where $\pi$ is the projection $\pi: M \rightarrow M^{*}$. The volume function

$$
V: M^{*} \rightarrow R
$$

is defined as follows :

$$
V\left(x^{*}\right)=\left\{\begin{array}{cl}
\operatorname{vol}(G(x)), & \text { if } G(x) \text { is a principal orbit } \\
m \cdot \operatorname{vol}(G(x)), & \text { if } G(x) \text { is an exceptional orbit } \\
0, & \text { if } G(x) \text { is a singular orbit }
\end{array}\right.
$$

where $m=$ the number of cosets in $K / H$ for $K$ an isotropy subgroup of the exceptional orbit and $H$ a principal isotropy subgroup. If we denote the union in $M$ of the principal orbits by $M_{(H)}$, it follows from the Slice Theorem that $V$ is continuous on $M^{*}$ and differentiable on $M_{(H)}^{*}$ [11]. Moreover, it is known that an orbit of maximal volume is a minimal submanifold of $M$ [10].

We will suppose that $f: M \rightarrow M$ is an equivariant isometry and study the geometric structure of periodic points and periodic orbits of $f$. It will be instructive to first examine two examples.

The following arise out of standard well-known examples; see, for example, [13, p. 23].

EXAMPLE 4.1. Let $G=S O(n)$ be the product action on $R^{n+1}=R^{n} \times$ $R$ where the action on the first factor is the standard orthogonal action and the action on the second factor trivial. Then $G$ acts on the unit sphere $M=S^{n}(1)$ of $R^{n+1}$ isometrically with 


$$
\begin{aligned}
M^{*} & =\left\{(x, y) \in R^{2}: x \geq 0, x^{2}+y^{2}=1\right\} \\
& =\left\{(\cos \theta, \sin \theta):-\frac{\pi}{2} \leq \theta \leq \frac{\pi}{2}\right\} \\
& \approx\left\{\theta:-\frac{\pi}{2} \leq \theta \leq \frac{\pi}{2}\right\}
\end{aligned}
$$

The volume function $V: M^{*} \rightarrow R$ is given by

$$
V(\theta)=\alpha_{n-1} \cos ^{n-1} \theta, \alpha_{n-1}=\text { volume } S^{n-1}(1) .
$$

$V$ has a unique maximum at $\theta=0$ with corresponding orbit $G\left(x_{1}\right) \approx$ $S^{n-1}(1)$ which is a principal orbit and a minimal submanifold of $M$. If now $f: M \rightarrow M$ is an equivariant isometry, it follows that $G\left(x_{1}\right)$ is invariant under $f$. If $n$ is odd, it follows from Fuller's Theorem that every point of $G\left(x_{1}\right)$ is a periodic point of $f$ since $\chi\left(G\left(x_{1}\right)\right)=\chi\left(S^{n-1}\right)=2$.

$V$ assumes minimum values at $\theta=-\frac{\pi}{2}$ and $\frac{\pi}{2}$ with corresponding orbits $G\left(x_{2}\right)$ and $G\left(x_{3}\right)$ which are fixed points of $G$. Hence $x_{2}$ and $x_{3}$ are periodic points of $f$.

EXAMPLE 4.2. Let $G=S O(p) \times S O(q)$ act diagonally on $R^{p} \times R^{q}$ via the standard orthogonal actions. The orbit at

$$
(x, y) \in R^{p} \times R^{q} \text { is } S^{p-1}(\|x\|) \times S^{q-1}(\|y\|) .
$$

$G$ induces an action of $G$ on $M=S^{n}(1), n=p+q-1$. The volume function $V$ is given by

$$
V(\|x\|,\|y\|)=\alpha_{p-1} \alpha_{q-1}\|x\|^{p-1}\|y\|^{q-1} .
$$

Hence $V$ has a maximum at $(\|x\|,\|y\|)$ where

$$
\|x\|^{2}=\frac{p-1}{p+q-2},\|y\|^{2}=\frac{q-1}{p+q-2}
$$

and minimums at $x=0$ and $y=0$. It follows, that if $f$ is an equivariant isometry on $M$ that $f$ has 3 periodic orbits, i. e. orbits which are invariant under some iterate of $f$ :

$$
G\left(x_{1}\right)=S^{p-1}\left(\sqrt{\frac{p-1}{p+q-2}}\right) \times S^{q-1}\left(\sqrt{\frac{q-1}{p+q-2}}\right),
$$

where $G\left(x_{1}\right)$ is a principal orbit, a minimal submanifold of $M$, and invariant under $f$.

$$
G\left(x_{2}\right)=S^{p-1}(1) \text { and } G\left(x_{3}\right)=S^{q-1}(1),
$$


which are singular orbits. Moreover $G\left(x_{2}\right)$ (respectively $G\left(x_{3}\right)$ ) is a minimal submanifold of $M$ if $p \neq 1$ (respectively $q \neq 1$ ).

If both $p$ and $q$ are odd, then every point in all the orbits $G\left(x_{i}\right), i=1$, 2,3 is a periodic point of $f$. If $n$ is even and either $p$ or $q$ odd, say $p$ odd, then every point of $G\left(x_{2}\right)$ is a periodic point of $f$. More generally we have the following result.

THEOREM 4.3. Let $G$ act on $M$ with principal orbits of codimension one with $M^{*}$ a closed interval. If $f$ is an equivariant isometry on $M$, there exists at least 3 periodic orbits $G\left(x_{i}\right), i=1,2,3$, such that:

i ) $G\left(x_{1}\right)$ is a principal orbit and a minimal submanifold of $M$.

ii ) $G\left(x_{2}\right)$ and $G\left(x_{3}\right)$ are singular orbits, each of which is either a minimal submanifold of $M$ or a fixed point of $G$.

iii) If $\chi\left(G\left(x_{i}\right)\right) \neq 0$ for some $i=1,2,3$ then every point in $G\left(x_{i}\right)$ is a periodic point of $f$.

iv) If $\chi(M) \neq 0$, either $G\left(x_{2}\right)$ or $G\left(x_{3}\right)$ is a minimal submanifold with each point a periodic point of $f$.

Proof. The volume function $V$ on $M^{*}$ assumes minimums at the 2 end points of $M^{*}$; hence (ii) follows.

Suppose $V$ has a maximum at $x^{*}$ with $V\left(x^{*}\right)=a$. Then $V^{-1}(a)$ is a closed subset of $M^{*}$. If $C_{1}, \cdots, C_{k}$ are the components of $V^{-1}(a)$, each $C_{i}$ is either a point or a closed subinterval $I_{i}$ of $M^{*}$. Since $\mathrm{f}$ is an isometry, it preserves the volume of each orbit and, hence, $f_{*}$, the induced mapping on $M^{*}$, must permute the $C_{i}$. It follows that for each $i$, there exists a positive integer $k_{i}$ such that

$$
f_{*}^{k_{i}}: C_{i} \rightarrow C_{i}, i=1,2, \cdots, k .
$$

If $C_{i}$ is a point, $f_{*}^{k_{i}}\left(C_{i}\right)=C_{i}$ and $\pi^{-1}\left(C_{i}\right)=G\left(x_{1}\right)$ is a periodic orbit of $f$. If $C_{i}=I_{i}, f_{*}^{k_{i}}$ has a fixed point $x_{1}^{*}$ on $C_{i}$ by the Brower fixed-point theorem. So again $G\left(x_{1}\right)$ is a periodic orbit of $f$ and clearly a principal orbit and minimal submanifold of $M$. This establishes ( $\mathrm{i}$ ). (iii) follows from the Fuller Theorem and (iv) from 3.3.

The same argument of 4.3 can be used to establish:

THEOREM 4.4. Let $G$ be a compact connected Lie group of isometries on a closed connected Riemannian $n^{-m a n i f o l d ~} M$. Let $a \in R$ be a non -zero critical value of the volume function on $M^{*}$ and write

$$
V^{-1}(a)=C_{1} \cup C_{2} \cdots \cup C_{k}
$$

as the union of its components. Suppose finally for some $i, 1 \leq i \leq k$, that 
$\chi\left(C_{i}\right) \neq 0$ and that the Lefschetz fixed point theorem is valid for $C_{i}$.

If $f$ is an equivariant isometry on $M$, there exists a periodic orbit $G\left(x_{i}\right), x_{i}^{*} \in C_{i}$, which is a minimal submanifold of $M$ and either a principal or exceptional orbit. Moreover, if $\chi\left(G\left(x_{i}\right)\right) \neq 0$, each point in $G\left(x_{i}\right)$ is a periodic point.

We return briefly to Examples 3.5 and 3.6. In 3.5 the volume function on $M^{*}$ is given by

$$
V\left(\theta_{1}, \cdots, \theta_{k}\right)=\alpha_{p_{1}-1} \cdots \alpha_{p_{k}-1} \sin ^{p_{1-1}} \theta_{1} \cdots \sin ^{p_{k}-1} \theta_{k} .
$$

Hence there exists a unique maximum at

$$
\theta_{1}=\cdots=\theta_{k}=\pi / 2 \text {. }
$$

The orbit is

$$
G(x)=S^{p_{1}-1}(1) \times \cdots \times S^{p_{k}-1}(1) \times K / H
$$

which is principal and a minimal submanifold of $M$. If $\chi(G(x)) \neq 0$, i. e. $p_{i}$ is odd for all $i$ and $\chi(K / H) \neq 0$, and $f$ is an isometry of $M$, each point of $G(x)$ is a periodic point of $f$.

In 3.6 the volume function on $M^{*}$ is given by $V\left(\theta_{1}, \cdots, \theta_{k}\right)=\alpha_{p_{1}} \cdots$ $\alpha_{p_{k}} \theta_{1}^{p_{1}} \cdots \theta_{k}^{p_{k}}$

where

$$
M^{*}=\left\{\left(\theta_{1}, \cdots, \theta_{k}\right): \theta_{i} \geq 0 \text { and } \theta_{1}^{2}+\cdot+\theta_{k}^{2}=1\right\} .
$$

So $V$ has a maximum at

$$
\theta_{i}^{2}=p_{i} / n, i=1, \cdots, k \text {, }
$$

and

$$
G(x)=S^{p_{1}}\left(\sqrt{\frac{p_{1}}{n}}\right) \times \cdots \times S^{p_{k}}\left(\sqrt{\frac{p_{k}}{n}}\right) \times K / H
$$

is the unique principal orbit of maximum volume which is, of course, a minimal submanifold of $M$. Again if $\chi(G(x)) \neq 0$, i. e. $p_{i}$ is even for all $i$ and $\chi(K / H) \neq 0$, and $f$ is an isometry of $M$, each point of $G(x)$ is a periodic point of $f$.

The following is a special case of Theorem 3.2 of [14].

THEOREM 4.5. Let $G$ be a toral group, i.e., compact connected abelian Lie group, of isometries acting on a closed connected Riemannian $n$-manifold $M, \chi(M) \neq 0$. Then, if $f: M \rightarrow M$ is an equivariant isometry, 


$$
f^{k}: F(G, M) \rightarrow F(G, M)
$$

has a fixed point for some $k \leq N[F(G, M)]$. Moreover

$$
N[F(G, M)] \leq N(M) .
$$

COROLlary 4.6. Let $M$ be a closed connected Riemannian n-manifold with $\chi(M) \neq 0$, and $Z$ an infinitesimal isometry of $M$ with $Y=\operatorname{Zero}(Z)$, the zero-set of $Z$. If $f$ is an isometry of $M$ with

$$
d f\left(Z_{x}\right)=Z_{f(x)} \text {, all } x \in M,
$$

then

$$
f^{k}: Y \rightarrow Y
$$

has a fixed point for some $k \leq N(Y)$. Moreover

$$
N(Y) \leq N(M) \text {. }
$$

PROOF. Let $G$ be the closure of the 1-parameter subgroup of $\operatorname{Isom}(M)$ generated by $Z$. Then $G$ is a toral group and

$$
F(G, M)=\operatorname{Zero}(Z)=Y \text {. }
$$

The following result may be found in $[12$, p. 63].

THEOREM 4.7. (Kobayashi) Let $M$ be a closed Riemannian manifold and $f$ an isometry of $M$. If $F$ denotes the fixed point set of $f$ and $L(f)$, the Lefschetz number of $f$,

$$
L(f)=\chi(F) .
$$

REMARK 4.8. The Fuller (1.1) and Kobayashi (4.7) results hold without the assumption of connectedness of the space. In the remainder of the paper, for technical reasons, we will not assume that our Riemannian manifold $M$ is connected, e.g. $M$ might consist of components of different dimensions.

We conclude this section with a result on the existence of common periodic points for commuting isometries, which appears to be new.

THEOREM 4.9. Let $M$ be a closed Riemannian manifold with $\chi(M) \neq 0$. If $f_{1}, f_{2}, \cdots, f_{r}$ are commuting isometries on $M$, then there exists $l \geq 1$ such that

$$
f_{i}^{l}(x)=x \text { for some } x \in M, 1 \leq i \leq r .
$$

Proof. By the proof of the Fuller Theorem (1.1), there exists 
$k_{1} \leq N(M)$ such that

$$
L\left(f_{1}^{k_{1}}\right) \neq 0 .
$$

Let

$$
F_{k_{1}}=F\left(f_{1}^{k_{1}}, M\right) .
$$

Now $\mathrm{F}_{k_{1}}$ is a Riemannian manifold $[12,5.1]$ and by the Kobayashi theorem (4.7),

$$
\chi\left(F_{k_{1}}\right)=L\left(f_{1}^{k_{1}}\right) \neq 0 .
$$

Since $f_{1}$ and $f_{2}$ commute,

$$
f_{2}: F_{k_{1}} \rightarrow F_{k_{1}}, \quad \chi\left(F_{k_{1}}\right) \neq 0 .
$$

By (1. 1) again, there exists $k_{2} \leq N\left(F_{k_{1}}\right)$ such that

$$
L\left(f_{2}^{k_{2}} \mid F_{k_{1}}\right) \neq 0 .
$$

Now $F\left(f_{2}^{k_{2}}, F_{k_{1}}\right)$ is a Riemannian manifold and

$$
F\left(f_{2}^{k_{2}}, F_{k_{1}}\right)=F_{k_{1}} \cap F_{k_{2}},
$$

where $F_{k_{2}}=F\left(f_{2}^{k_{2}}, M\right)$ so by (4.7) again,

$$
\chi\left(F_{k_{1}} \cap F_{k_{2}}\right)=\chi\left[F\left(f_{2}^{k_{2}}, F_{k_{1}}\right)\right]=L\left(f_{2}^{k_{2}} \mid F_{k_{1}}\right) \neq 0 .
$$

Since $f_{1}, f_{2}$, and $f_{3}$ commute,

$$
f_{3}: F_{k_{1}} \cap F_{k_{2}} \rightarrow F_{k_{1}} \cap F_{k_{2}}
$$

and, proceeding in this way, we obtain $k_{3}, \ldots, k_{r}$ and the associated fixed point sets $F_{k_{3}}, \cdots, F_{k r}$. Finally let

$$
l=l c m\left(k_{1}, k_{2}, \ldots, k_{r}\right)
$$

and

$$
x \in F_{k_{1}} \cap F_{k_{2}} \cap \cdots \cap F_{k r},
$$

which is non-empty since

$$
\chi\left(F_{k_{1}} \cap F_{k_{2}} \cap \cdots \cap F_{k_{r}}\right) \neq 0 .
$$

CONJECTURE 4.10. We can choose

$$
l \leq[N(M)]^{r}
$$

in 4.9.

REMARK 4.11. If $f_{1}, f_{2}, \cdots, f_{r}$ in 4.9 lie in the same maximal torus $T$ 
of $\operatorname{Isom}(M)$, it follows immediately that they have a common fixed point, i. e., $l=1$. This is a consequence of the fact that if a toral group $T$ acts on $M, \chi[F(T, M)]=\chi(M)$; see for example [12,5.5(1)].

We observe that the Fuller theorem also holds for compact complex manifolds with self-holomorphic diffeomorphisms using the Dolbeault cohomology and the holomorphic Lefschetz fixed point theorem. More generally, we can obtain Fuller type theorems using cohomology of elliptic complexes and the Lefschetz fixed point theorem of Atiyah-Bott [1]. Hence we can show that if a toral group $T$ acts effectively and smoothly on a closed connected smooth manifold $M$ with signature, Sign $(M) \neq 0$, and if $f: M \rightarrow M$ is an equivariant diffeomorphism, then $f$ has a periodic point on $F(T, M)$.

\section{References}

[1] M. F. ATIYAh, and R. BotT, A Lefschetz fixed point formula for elliptic complexes. I, Ann. of Math. 86 (1967), 374-407.

[2] A. BOREL, Cohomology des Espaces Localement Compacts d'áprès J. Leray, Lecture Notes in Math. No. 2, Springer-Verlag, 1964.

[ 3 ] A. BoREL, et al., Seminar on Transformation Groups, Annals of Mathematics Studies 46, Princeton Univ. Press, 1960.

[4] Glen E. BREDON, Introduction to Compact Transformation Groups, Academic Press, New York and London, 1972.

[5] R. F. BRown, The Lefschetz Fixed Point Theorem, Scott, Foresman and Company, Glenview, Illinois, 1971.

[6] E. FADELL, Recent results in the fixed point theory of continuous maps, Bull. Amer. Math. Soc. 76 (1970), 10-29.

[ 7 ] F. B. FULLER, , The existence of periodic points, Ann. of Math. 57 (1953), 229-230.

[ 8 ] Werner Greub, Stephen HALPERIN, and Ray VAnstone, Connections, Curvature, and Cohomology, Vol. I, Academic Press, New York and London, 1972.

[ 9] Benjamin HALPERN, Fixed points for iterates, Pacific J. Math. 25 (1968), 255-275.

[10] W. Y. HSIANG, On compact homogeneous minimal submanifolds, Proc. Nat. Acad. Sci. USA 56 (1966), 5-6.

[11] W. Y. HSIANG, and H. B. LAWSON, Minimal submanifolds of low cohomogeneity, J. Diff. Geometry 5 (1971), 1-38.

[12] S. KoBAyASHI, Transformation Groups in Differential Geometry, Springer-Verlag, 1972.

[13] H. B. LAwSON, Lectures on Minimal Submanifolds, Vol. I, Publish or Perish, Inc, 1980.

[14] L. N. MAnN, Periodic points and invaraint orbits of equivariant maps, Mem. Fac. Sci. Kochi Univ. (Math.) 14 (1993), 15-21.

[15] James W. VICK, Homology Theory, Academic Press, New York and London, 1973.

Department of Mathematics and Statistics University of Massachusetts at Amherst Amherst, Massachusetts 01003 USA 